\title{
The Relationship between Emotional Intelligence and Socioeconomic status of caregivers on the Quality of Life in patient's with Alzheimer's dementia
}

\author{
Asira Chirmuley \\ ${ }^{1}$ Post Graduate Student, \\ Department of Psychology, Maniben Nanavati Women's College, Mumbai \\ E-mail - asira.richie@gmail.com
}

\section{ABSTRACT}

Dementia of Alzheimer's type (DAT) is the most common cause of dementia in the older people. The importance of Emotional Intelligence is in equipping caregivers to deal efficiently with Alzheimer patients. The current study seeks to evaluate the role played by Emotional Intelligence and Socio-economic Status of caregivers of Alzheimer patients on the Quality of Life of Alzheimer patients. The total numbers of participants in the study were 60 caregivers of Alzheimer patients who were given tests of Emotional Intelligence scale and Alzheimer's disease Related Quality of Life (ADRQoL). The data was analysed using appropriate statistical methods. The Emotional Intelligence and the socioeconomic status of caregivers of Alzheimer patients had no significant effect on the Quality of Life of Alzheimer patients and major statistical significance was noted. Further enquiry into other factors affecting the Quality of Life of Alzheimer patients is warranted.

Key words: Alzheimer's dementia, dementia, quality of life, emotional intelligence, socioeconomic status, caregivers.

\section{INTRODUCTION}

Dementia (now called as major neurocognitive disorder according to DSM V) is a syndrome characterised by progressive and usually irreversible neurocognitive deficits in one or more cognitive domains such as memory, language, judgment, planning, and social interaction. Dementia impacts personal, family, and societal life. It reduces life span and induces caregiver's strain at family level. Dementia of Alzheimer's type (DAT) is the most common cause of dementia in the older people (72\%) [1].The severity of the disorder is graded depending on the increasing dependence on the caregivers with mild cases requiring least assistance and severe cases fully dependent on the caregivers. Management of DAT involves two principle strategies, pharmacological and non pharmacological. As the outcomes of pharmacologic treatment are inconsistent, considering the alternative (non pharmacologic) strategies (communication methods, therapeutic activities, environment modification and maintaining overall health) in the management becomes imperative [2].

Emotional intelligence (EI) is the ability to recognize one's own and other people's emotions, to discriminate between different feelings and label them 
appropriately, and to use emotional information to guide thinking and behaviour [3]. In the ability model, emotional intelligence is said to have four dimensions (a) perceiving emotions, (b) using emotions to facilitate thought, (c) understanding emotional information, and (d) managing emotions [4].The importance of Emotional Intelligence is in equipping caregivers to deal efficiently with Alzheimer patients. Caregivers of patients fight their own battle while caring for the patient. Alzheimer's disease is incurable, progressive in nature and goes on worsening at a fast pace. Such helpless scenarios take a toll on the caregiver's mental health [5].Stress and psychological morbidity is often higher in caregivers of Alzheimer's patients. Behavioral symptoms and impairment in instrumental activities of daily living are found to be associated with caregiver stress. Length of care is also associated with caregiver psychological morbidity [6]. To help them deal with these unfortunate realities of the disorder, Emotional intelligence training or interventions on caregivers would prove very beneficial. The ultimate measures of success of such a treatment approach are improved Quality of life, delayed institutionalization, slowed rate of progression of the disease, people who achieve their potential and reduced need for medication [2]. These interventions could only be achieved with the help of caregivers. This research aims to execute this study in order to understand and weigh the role played by Caregiver's Emotional Intelligence on the recovery of Alzheimer patients. A critical evaluation of literature with Implications for mental health nursing leadership has argued that emotional intelligence (EI) is necessary for the development of interpersonal and professional competence in nurses [7]. Correlations between emotional intelligence and better overall health, increased work satisfaction, higher spiritual well-being, and decreased risk of job burnout were noted [7]. Apart from the emotional intelligence socio-economic status is also an important determinant as it correlates with the level of care that can be afforded by the caregiver for the patient such as better pharmacologic as well as non-pharmacological care. The study concentrates on caregivers with middle class of income that is, higher middle class and lower middle class. According to India's National Council of Applied Economic Research (NCAER), NCAER's current definition identifies the middle class as comprising of two sub-groups: 'seekers' with annual household income between Rs. 2,00,000 and Rs. 5,00,000, and 'strivers' with annual household income between Rs. 5,00,000 and Rs. 1 million [8]. Good Quality of life of caregivers is associated with the improved quality of life in Alzheimer's patients [9-10]. The current study seeks to evaluate the role played by Emotional Intelligence and Socio-economic Status of caregivers of Alzheimer patients on the Quality of Life of Alzheimer patients.

\section{METHODOLOGY}

\section{Participants}

The total numbers of participants in the study were 60 caregivers of Alzheimer patients. DSM IV TR criteria and MRI Findings were used to diagnose the patients of Alzheimer's disease in the patients from psychiatry department and the same patients were then referred to the neurology department of the tertiary general hospital for confirmation of the diagnosis. The data was gathered by way of purposive sampling. Inclusion criteria were 1) Patients diagnosed as having Dementia of Alzheimer's type as per DSM-IV-TR criteria and 2) Caregivers belonging to Age 18-60 yrs. Exclusion criteria were 1) Patients with Medical/ Psychiatric co-morbidity and 2) Patients/Caregivers not willing to give Informed Valid Consent. The study involved one demographic characteristic as an experimental variable 'Socio-economic status'. 


\section{Procedure}

Caregivers of Alzheimer patients were given tests of Emotional Intelligence scale [11] and Alzheimer's disease related Quality of Life scale [12]. The experimental variables in the study were Emotional Intelligence and Socioeconomic status of caregivers of Alzheimer patients. Emotional Intelligence of caregivers of Alzheimer patients has three levels - High and Low. And, Socioeconomic status of caregivers of Alzheimer patients has two levels - Higher Middle Class and Lower Middle Class. The dependent variable in the study was - Quality of Life of Alzheimer patients as measured by their scores on Alzheimer's Disease Related Quality of Life. The data obtained was manually scored using Emotional Intelligence Scale (EIS) and Alzheimer's disease Related Quality of Life (ADRQL) scoring methods available in their respective manuals.

\section{Scales used}

The test used in the study to examine the Emotional Intelligence of caregivers of Alzheimer patients was Emotional Intelligence Scale (EIS) [11]. The test has in all 34 items and measures 10 factors. It's a Likert scale as it obtains participants' preferences or degree of agreement with a statement or set of statements. Categories for Emotional Intelligence according to the score obtained were: 1) High - 85 and above 2) Normal - 52-84 3) Low - 51 and below. The split-half reliability coefficient as calculated on the sample of 200 subjects was found to be 0.88 . In order to find out the validity from the coefficient of reliability, the reliability index was calculated, which indicated high validity on account of being 0.93 .

The revised 40-item version of the ADRQL was used [12]. Categories for Alzheimer's Disease Related Quality of Life (ADRQL) were: 1) Social Interaction, 2) Awareness of Self, 3) Feelings and mood, 4) Enjoyment of activities. Overall, the revised ADRQL has good internal consistency. Cronbach's alpha was 0.86. The revised ADRQL exhibited good internal consistency across all three settings: nursing home (0.79), community (0.87), and assisted living (0.84). In a sub-sample of community residents, the revised ADRQL was able to discriminate between individuals with severe cognitive impairment (MMSE < 18) and other individuals, and between individuals with and without behavioral and psychological symptoms. The overall revised ADRQL score was able to discriminate between persons with $A D L$ difficulties and those with no ADL difficulties.

\section{Statistical Analysis}

The data obtained from the above method was pooled and subjected to the statistical analysis using SPSS software. SPSS trial version 16.0 for Windows 8 was put to use. The statistical method that was employed in the study was Analysis of Variance. The purpose for doing ANOVA is to find if there is any difference between groups on some variable that is, to infer whether there are real differences between the means of three or more groups or variables in a population, based on sample data [13]. The analysis of variance can be presented in terms of a linear model, which makes following the assumptions about the probability distribution of the responses Independence of observations, Normality, Equality [14]. This study has used Two-way Analysis of Variance because of the experimental design (two independent variables and one dependent variable). 


\section{RESULTS}

The current study was undertaken to examine the relationship between the Emotional Intelligence and the Socio-economic status of caregivers of Alzheimer patients on the Quality of Life of Alzheimer patients. The Independent variables employed in the study were Emotional Intelligence (High, Low) of caregivers of Alzheimer patients and Socio-economic status of caregivers (Upper Middle class, Lower Middle Class) of Alzheimer patients. The dependent variable in the study was Alzheimer's disease related Quality of life.

\section{Descriptive Statistics for Variables}

The range, means, and standard deviation, skewness and kurtosis for each variable in the study are reported in Table 1.

\section{Variable 1 - Emotional Intelligence}

The mean for High Emotional Intelligence (level 1) was 49.47 and standard deviation was 1.875. Skewness for High Emotional Intelligence was found to be 0.012 , which indicates normal distribution. Kurtosis was found to be -0.956 . The mean for Low Emotional Intelligence (Level 2) was 62.03 and standard deviation was 1.695. Skewness for Low Emotional Intelligence was found tobe - 0.12 which shows non-normal distribution. Kurtosis was found to be 0.372 .

\section{Variable 2 - Socio-economic status}

The mean for Upper Middle class (Level 1) was 61.56 and Standard deviation was 1.685. Skewness for Upper Middle Class was -0.141 which implies non-normal distribution. Kurtosis was found to be 0.312 . The mean for Lower Middle class was 49.52 and standard deviation was 1.907. Skewness for Lower Middle Class was 0.004 which indicates normal distribution. Kurtosis was found to be -1.030 .

\section{Two-way Analysis of Variance}

Two-way Analysis of Variance was performed on the sample of 60 participants. It was conducted to compare the effect of Emotional Intelligence and Socioeconomic status on Alzheimer's disease related Quality of Life. The results depicted in Table 3, confirmed that Emotional Intelligence and Socio-economic status of caregivers of Alzheimer patients had no effect on the Quality of Life of Alzheimer patients. That is, the results were found to be non-significant at 0.05 level $[\mathrm{df}=(59,1), \mathrm{F}=0.007,0.582]$. 


\section{Normal Q-Q Plot of ADRQL_Percentage_}

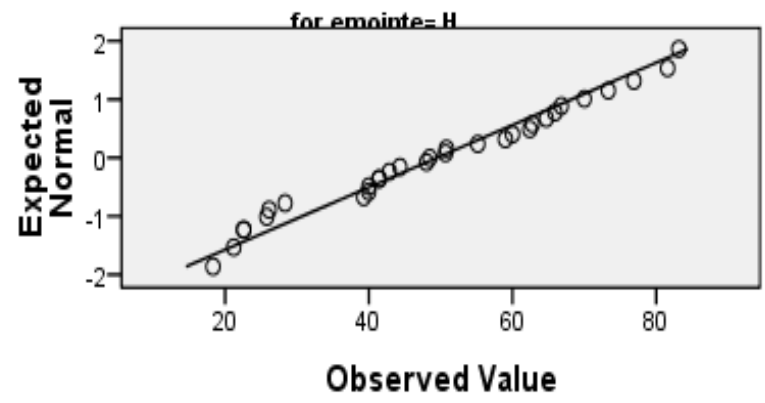

Figure 2.1 - Normal Q-Q Plot for High Emotional Intelligence Normal Q-Q Plot of ADRQL_Percentage_

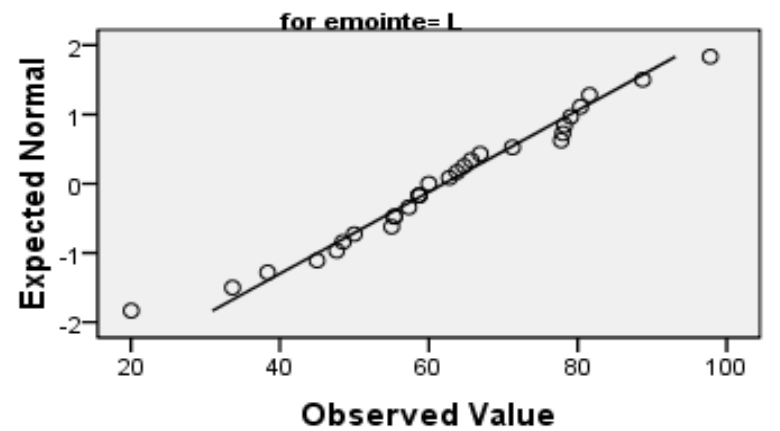

Figure 2.2 - Normal Q-Q plot for Low Emotional Intelligence Normal Q-Q Plot of ADRQL_Percentage_

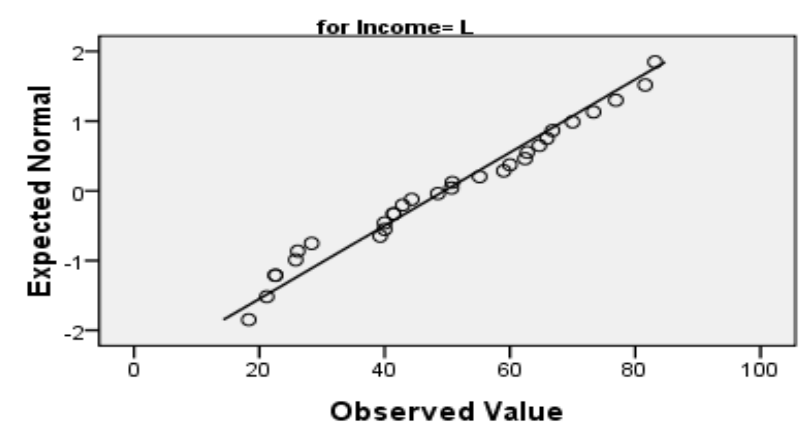

Figure 2.3- Normal Q-Q plot for Lower Middle Class

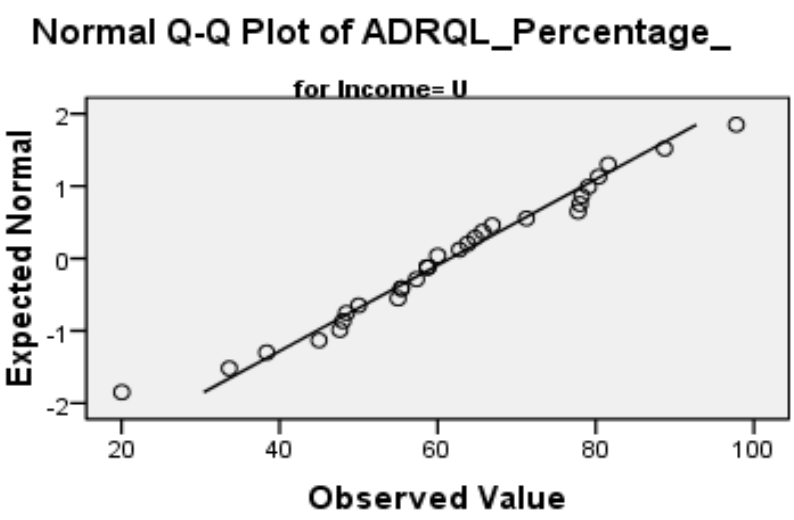

Figure 2 - Normal Q-Q plot for Upper Middle Class 


\begin{tabular}{|llllll|} 
Source & $\begin{array}{l}\text { Sum of } \\
\text { Squares }\end{array}$ & df & $\begin{array}{l}\text { Mean } \\
\text { square }\end{array}$ & F & Sig. \\
\hline Income & $2364.987^{\mathrm{a}}$ & 2 & 1182.493 & 3.624 & 0.033 \\
Emo. Int. & 57828.435 & 1 & 57828.435 & 177.251 & 0.000 \\
Income* & 2.191 & 1 & 2.191 & 0.007 & 0.935 \\
Emo.Int. & 189.840 & 1 & 189.840 & 0.582 & 0.449 \\
Error & 0.000 & 0 & & & \\
Total & 18596.348 & 57 & 326.252 & & \\
Corrected & 206080.600 & 60 & & & \\
Total & 20961.335 & 59 & & & \\
\hline
\end{tabular}

ANOVA Statistics

\section{DISCUSSION}

According to a research study a close relationship is positively related to the progression of Alzheimer's disease. The study showed that having a close caregiver, especially a spouse, resulted in a slow decline of Alzheimer's. The findings of the above study showed that the Quality of relationship of caregivers with Alzheimer patients may play a vital role in the recovery of Alzheimer patients. The results of the above study are in contrast with the current study which did not evaluate the Quality of relationship of caregivers with the Alzheimer patients [15].

Another research conducted on Quality of life for caregivers of people with Alzheimer's disease [16] showed that the Alzheimer's disorder caregivers' Quality Of Life was influenced by their patients Quality Of Life and that the caregivers identified several factors that affect their own Quality Of Life suggesting the importance of conducting intervention research for caregivers in countries where this has not yet been performed. The international literature reports that educational interventions improve caregivers Quality Of Life [16].

In another research authors showed that higher emotional intelligence is associated with better overall health, increased work satisfaction, higher spiritual well-being, and decreased risk of job burnout in the mental health nursing staff. This is expected to translate into betterment of quality of life of the patients however the current study suggests otherwise [17].

The current research finding also found no significant relationship between Socio-economic status of caregivers of Alzheimer patients and the Quality of Life of Alzheimer patients. This could be due to the loosely defined income categorization. Moreover, due to the non-significant results, an interaction effect could not be established. The qualitative observations of the study derived from the detail, informal case discussions with the caregivers of Alzheimer patients were that, in the case of most of the patients, there was a retirement syndrome observed that is, most of the patients after their retirement did not engage in any mentally demanding activity which then left their mind idle, doing nothing constructive. This pattern was seen in almost half of the cases. It is still unclear whether this has anything to do with the onset of Alzheimer's but it might be playing a role in deteriorating the quality of life which needs to be further studied and explored.

Another observation was that most of the patients had a significant amount of disengagement with the society in general and this was due to varied reasons such as health concerns, unhealthy home atmosphere, age, etc. This isolation from the 
society could also play role in determining the quality of life of the patient. Moreover, support system, social circle, emotional difficulties also might have some role to play in the course of Alzheimer's disorder. As the case discussions with the caregivers were not scientifically conducted and studied, these ancillary observations should be taken as factors that could have affected the Quality of Life of Alzheimer's disease patient. Further detail, scientific analysis of case studies is necessary to come to any conclusion regarding the same.

The research would add to the understanding of the relationship between the variables under study. However, certain limitations of the study need to be acknowledged. The scale that was used to assess the Emotional Intelligence of caregivers of Alzheimer patients could have been a more standardized scale on a greater number of populations. That is, a more standardized scale could have been employed in the study to assess Emotional Intelligence of caregivers of Alzheimer patients. This would have eliminated the influence of social desirability while filling in the Emotional Intelligence Scale. A larger data should have been collected. This could have given a more detailed understanding of the relationship between the variables under study and would have been a better representation of the sample. The scale that was used to examine the Quality of Life of caregivers of Alzheimer patients was not standardized on Indian population. In addition to studying the Socio-economic status of caregivers of Alzheimer patients, the quality of treatment interventions, stress and depression in the caregivers should also be taken into consideration. In India the income categories are loosely defined. There are not enough consensuses on the income brackets for Upper Middle Class and Lower Middle Class. This could have negatively impacted the variable under study, the Socio-economic status of caregivers of Alzheimer patients.

From a theoretical perspective, there is a dearth of quantitative research in the area of factors affecting the Quality of Life of Alzheimer patients in India. As a result, a strong foundational support was not sought. This could have played a vital role in the studying the variables in the present study in more detail and applying them to Indian population. Future research might benefit from a larger data and use of a better instrument to examine Emotional Intelligence and consideration of factors like the quality of treatment interventions, stress and depression in caregivers, awareness about caregiving with respect to Alzheimer's disorder, coping interventions for caregivers of Alzheimer patients, Quality of Life of caregivers of Alzheimer patients, money spent on treatment by caregivers, caregiver issues etc. The importance of qualitative research in bringing understanding of the essence of caregiving of persons with Alzheimer's disease is well-documented. Qualitative research methodologies such as hermeneutic phenomenology, descriptive studies with focus groups, and individual interviews to identify needs and concerns of caregivers of Alzheimer's disease patients which include the need for knowledge about the disease, care recipient and caregiver issues, resource needs, and coping post-nursing home placement can be undertaken [17].

The findings of the present study have applicability to varied areas such as Caregiver institutions, Family caregivers association, mental health professionals, Social workers, Psychiatrists, Neurosurgeons, and Neuropsychologists. The results of the present study found no significant relationship between Emotional Intelligence and Socio-economic status of caregivers of Alzheimer patients on the Quality of Life of Alzheimer patients. This might indicate that there are other factors for improving or enriching the Quality of Life of Alzheimer patients than Socio-economic status and Emotional Intelligence. This could prove to be beneficial to mental health professionals who are working on preventing and treating disorders like Alzheimer's especially, with the use of caregiving. Moreover, Caregiver training for Neurodegenerative disorders like 